\title{
STING activation by cytoplasmic mtDNA triggers renal inflammation and fibrosis
}

CS

\section{The mtDNA} is recognized as foreign, which leads to activation of the innate immune system.

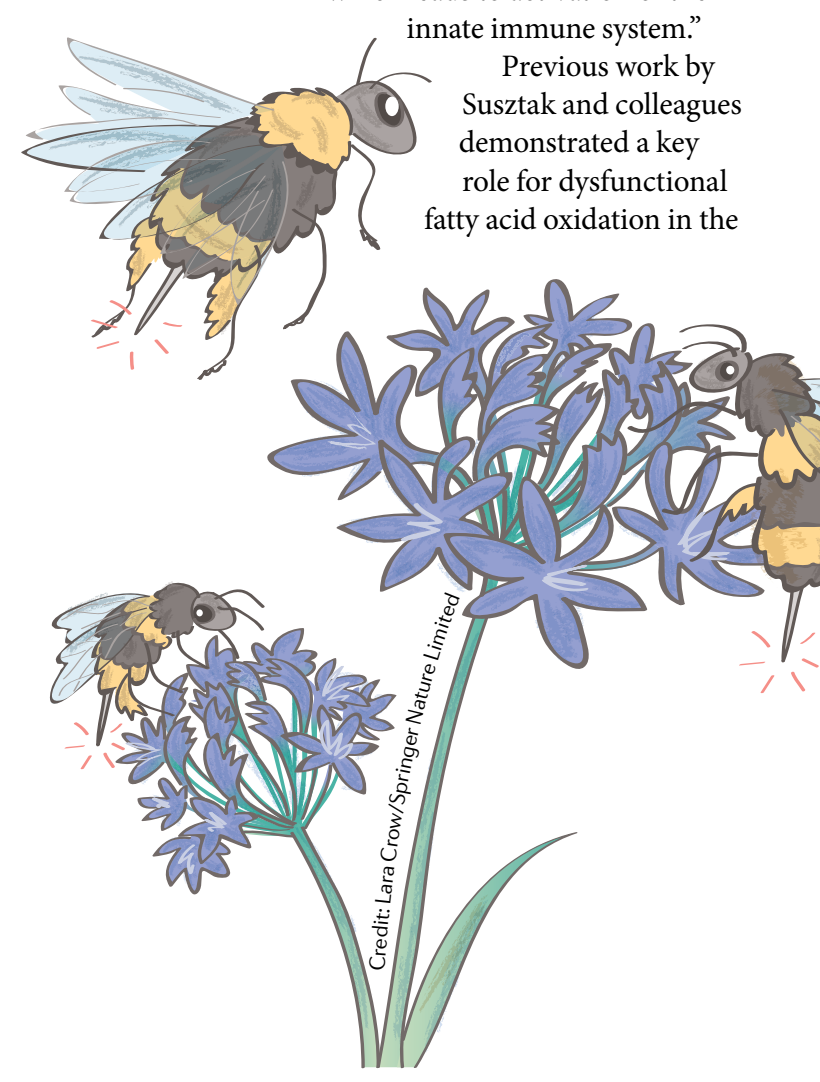

Mitochondrial dysfunction and inflammation have both been linked to fibrotic kidney diseases; however, the relationship between these two entities is not well understood. Research now shows that recognition of dysfunctional mitochondrial DNA (mtDNA) in the cytosol of tubule epithelial cells (TECs) by the cGAS-stimulator of interferon genes (STING) sensing pathway induces an immune response that triggers fibrotic changes. "We show that loss of mitochondrial integrity not only leads to kidney disease by inducing a cellular energy deficit, but also by promoting the release of mtDNA into the cytosol," explains Katalin Susztak. "In this instance, the mtDNA is recognized as foreign, which leads to activation of the te immune system" agues demonstrated a key role for dysfunctional fatty acid oxidation in the development of renal fibrosis. To investigate the pathways that control TEC metabolism and the consequences of their dysregulation, the researchers analysed gene and protein expression in kidney tubules from humans and animal models with fibrosis. Overall, they observed a significant reduction in levels of mitochondrial genes and proteins in the fibrotic samples. In particular, levels of the mitochondrial transcription factor A (TFAM), which has a key role in the maintenance of mtDNA, correlated positively with estimated glomerular filtration rate and negatively with the extent of fibrosis. Notably, levels of inflammatory cytokines also correlated with the extent of fibrosis.

To model the metabolic defect observed in murine and human fibrotic kidneys, Susztak and colleagues generated tubule-specific Tfam-knockout mice. These mice showed disorganization and loss of mitochondria with accompanying energy deficits by 6 weeks of age, and kidney fibrosis by 12 weeks of age. Apoptosis of tubule cells was observed in 12-week-old mice and correlated with the onset of fibrosis, but not with the metabolic changes. Use of RNA sequencing to delineate the cellular pathways affected by TFAM deficiency showed that kidneys of tubule-specific Tfam-knockout mice had lower expression of mitochondrial-encoded genes and increased expression of genes involved in immune and inflammatory pathways compared with levels in control kidneys. A time-course analysis indicated that kidneys of Tfam-knockout mice experience significant upregulation of inflammatory cytokine expression between 6 and 12 weeks of age, which coincides with the onset and progression of fibrotic changes.

To examine the effects of TFAM depletion on inflammatory pathways, the researchers examined primary cultures of TECs from kidneys of tubule-specific Tfam-knockout and control mice. TECs from Tfamknockout mice showed evidence of an early interferon response involving activation of $\mathrm{NF \kappa B}$, with subsequently increased expression of genes encoding proinflammatory cytokines. The researchers also observed evidence of disorganized mtDNA architecture and leakage of this mtDNA into the cytosolic fraction, suggesting a role for the cytosolic DNA sensor, cGAS-STING, in initiating the immune response. In support of this hypothesis, silencing of cGAS and STING in cultured cells did not affect the mitochondrial changes, but reduced the expression of inflammatory genes. In line with these in vitro findings, genetic and pharmacologic inhibition of STING reduced inflammation, TEC apoptosis and fibrosis in tubule-specific Tfamknockout mice. Moreover, genetic and pharmacologic inhibition of STING also attenuated renal fibrosis in mice with folic-acid-induced kidney injury.

Susztak believes her findings could have significant therapeutic potential, particularly given that multiple STING inhibitors have been developed. "These inhibitors could be used to treat fibrotic kidney disease," she says. "Our group is very interested in understanding how metabolic and inflammatory changes in proximal tubules contribute to kidney disease development, and would be keen to see these agents tested therapeutically".

Susan J. Allison

ORIGINAL ARTICLE Chung, K. W. et al. Mitochondrial damage and activation of the STING pathway lead to renal inflammation and fibrosis. Cell Metab. https://doi.org/10.1016/ .cmet.2019.08.003 (2019) 